\title{
Establishment of the circadian metabolic phenotype strategy in spontaneously hypertensive rats: a dynamic metabolomics study
}

Huanjun Wang ${ }^{1} \mathbb{C}$, Xiaoming Wang ${ }^{2,3,4}$, Dongmei Qi ${ }^{2,3,4}$, Mengjia Sun ${ }^{1}$, Qingqing Hou' ${ }^{1}$ Y Yunlun $\mathrm{Li}^{\mathrm{i}^{*}}$ and Haiqiang Jiang ${ }^{2,3,4^{*}}$

\begin{abstract}
Background: Circadian rhythms play a fundamental role in the progression of cardiovascular events. Almost all cardiovascular diseases have a circadian misalignment usually characterized by changes in metabolites. This study aimed to dynamically monitor rhythmic biomarkers, to elucidate the metabolic pathways that are potentially under circadian control in spontaneously hypertensive rats (SHRs), and to eventually establish a circadian metabolic phenotype strategy based on metabolomics.

Methods: In this study, an untargeted metabolomics technology was used to dynamically monitor changes in serum metabolites between SHR model group and WKY control group. Liquid chromatography-mass spectrometry (LC-MS) combined with multivariate statistical analysis was applied to identify markers of hypertension rhythm imbalance. The concentrations of amino acids and their metabolites identified as markers were quantified by a subsequent targeted metabolomics analysis. Overall, these approaches comprehensively explored the rhythm mechanism and established a circadian metabolic phenotype strategy.

Results: The metabolic profile revealed a disorder in the diurnal metabolism pattern in SHRs. Moreover, multivariate statistical analysis revealed metabolic markers of rhythm homeostasis, such as arginine, proline, phenylalanine, citric acid, L-malic acid, succinic acid, etc., accompanied by an imbalance in hypertension. The key metabolic pathways related to rhythm imbalance in hypertension were found by enrichment analysis, including amino acid metabolism, and the tricarboxylic acid cycle (TCA). In addition, the quantitative analysis of amino acids and their metabolites showed that the changes in leucine, isoleucine, valine, taurine, serine, and glycine were the most obvious.

Conclusions: In summary, this study illustrated the relationship between metabolites and the pathways across time on hypertension. These results may provide a theoretical basis for personalized treatment programmes and timing for hypertension.
\end{abstract}

\footnotetext{
*Correspondence: li.yunlun@163.com; jhq12723@163.com

${ }^{4}$ Shandong Provincial Key Laboratory of Traditional Chinese Medicine

for Basic Research, Shandong University of Traditional Chinese Medicine,

Jinan 250355, People's Republic of China

${ }^{5}$ TCM Clinical Research Base for Hypertension, Affiliated Hospital

of Shandong University of Traditional Chinese Medicine, Jinan 250011,

People's Republic of China

Full list of author information is available at the end of the article
}

\section{$\triangle B M C$}

Ther(s) 2020. This article is licensed under a Creative Commons Attribution 4.0 International License, which permits use, sharing, adaptation, distribution and reproduction in any medium or format, as long as you give appropriate credit to the original author(s) and the source, provide a link to the Creative Commons licence, and indicate if changes were made. The images or other third party material in this article are included in the article's Creative Commons licence, unless indicated otherwise in a credit line to the material. If material is not included in the article's Creative Commons licence and your intended use is not permitted by statutory regulation or exceeds the permitted use, you will need to obtain permission directly from the copyright holder. To view a copy of this licence, visit http://creativeco mmons.org/licenses/by/4.0/. The Creative Commons Public Domain Dedication waiver (http://creativecommons.org/publicdomain/ zero/1.0/) applies to the data made available in this article, unless otherwise stated in a credit line to the data. 
Keywords: Circadian rhythm, Hypertension, Metabolomics, Amino acids

\section{Background}

Hypertension is a common cardiovascular disease characterized by a persistent increase in arterial blood pressure, which contributes to cardiovascular morbidity and mortality. Although hypertension has a high incidence in the population, there is no effective treatment for this disease; thus high blood pressure is known as a "silent killer" [1]. In recent years, hypertension, as one of the typical cardiovascular diseases, has been investigated in a series of studies using spontaneously hypertensive rats (SHRs) as model animals. Several biomarkers were previously found in a metabolomics study by this research group. These biomarkers were mainly associated with lipid, vitamin and amino acid metabolism [2, 3]. However, in these experimental studies of hypertension, changes in metabolites were measured at one time point, and dynamic monitoring was not performed. In addition, the circadian clock represents a key timekeeping mechanism for many fundamental physiological processes such as the cell cycle and metabolism [4]. As such, the determination of the mechanisms underlying hypertensive metabolic disorders at a fixed time is not a comprehensive approach. In general, the rhythmic oscillation of organisms naturally changes. Nearly all aspects of physical behaviours change over time, including light/ dark, sleep/wakefulness, food intake/fasting, digestion, detoxication, and blood pressure $[5,6]$.

Animals possess an internal molecular mechanism called the "circadian clock", in which the endogenous, self-sustained oscillations over a period of approximately $24 \mathrm{~h}$ manifest in diverse physiological and metabolic processes [7]. Owing to metabolites oscillation throughout a day, clinical sampling at a single time point can easily lead to false or misleading diagnoses and even treatment outcomes $[8,9]$. Circadian rhythms are generated by master clock neurons in the suprachiasmatic nucleus $(\mathrm{SCN})$, which are critical in the physiology and behaviour of most organisms. The SCN and its efferent targets integrate light and feeding signals to entrain behavioural rhythms and to regulate clock cells in peripheral tissues, including the liver, adipose tissue and muscle [10]. Therefore, metabolic disorders are often associated with imbalanced circadian rhythms over time, resulting in many pathophysiological conditions such as obesity [11], metabolic syndrome, type 2 diabetes [12], and cancer [13]. The circadian rhythm is highly correlated with metabolites. The study of the circadian rhythm can reflect temporal changes in metabolites, which contributes to the comprehensive interpretation of a disease $[6,14]$. To determine the underlying mechanism linking hypertension to metabolic disorders, it is essential to understand the temporal changes in differential metabolites, that is, the changes in metabolites under the influence of circadian rhythm. Previous research has found circadian rhythms of obesity, diabetes, hyperlipidaemia and other chronic diseases through metabolomics studies [11, 12, 14]; however, research on the rhythm of hypertension has not been reported. To discover rhythmic biomarkers and elucidate the metabolic pathways that are potentially under circadian control in SHRs, a circadian metabolic phenotype strategy based on metabolomics was established. In the present study, it was hypothesized that the metabolites associated with hypertension are abnormal within 1 day. Therefore, serum samples were collected from SHRs and Wistar-Kyoto Rats (WKYs) at multiple time points to compare metabolite changes. The entire research process was based on a liquid chromatography-mass spectrometry (LC-MS) metabolomics platform and amino acid analysis. By comparing the serum metabolites over a day, we found biomarkers and pathways associated with rhythmic changes in SHR model. Our results highlight the links between time and metabolites.

\section{Materials and methods}

\section{Animals}

All animal studies were approved by the Animal Ethics Committee of Shandong University of Traditional Chinese Medicine and all experimental procedures were performed in strict accordance with the international regulation of animal welfare. Nine-week-old male SHRs $(215 \pm 10 \mathrm{~g})$ and WKYs $(210 \pm 10 \mathrm{~g})$ were purchased from Beijing Vital River Laboratory Animal Technology Co, Ltd. (certificate of conformity: SCXK (Beijing) 20160006). Before the experiment, all the rats were adaptively fed for 1 week. The SHRs were considered the model group, and the WKYs were considered the control group. Six male rats from the model group and from the control group were randomly assigned to each time point (a total of 13 points) point maintained on a $12 \mathrm{~h}$ light $/ 12 \mathrm{~h}$ dark cycle with standard chow and water provided ad libitum for 8 weeks. Afterwards, their blood pressure was measured by a BP-600 non-invasive tail artery blood pressure measurement system (Chengdu Techman Software Co., Ltd., Chengdu, China). Body weight was measured weekly. The volume of water and the weight of chow consumed by the rats in every cage were measured daily. Moreover, face temperature and pain threshold were also measured in the model and control groups by 
a SW-200 Photothermal Tail Pain Tester (Chengdu Techman Software Co., Ltd., Chengdu, China) and a GM300 Infrared Thermometer (Shenzhen Jumaoyuan Science and Technology Co., Ltd., Shenzhen, China), respectively. After 8 weeks, blood from the inferior vena cava was immediately collected after intraperitoneal injection of $1.5 \%$ sodium pentobarbital anaesthesia at a dose of $30 \mathrm{mg} \cdot \mathrm{kg}^{-1}$.

\section{Chemical and materials}

Acetonitrile (HPLC grade) was purchased from Fisher Scientific (Thermo Fisher, CA, USA); formic acid was purchased from Fisher Scientific (Thermo Fisher, CA, USA); water was obtained from Watson's (Watsons Food and Beverage Co., Ltd., Guangzhou, China); and the amino acid mixture standard solution, type AN-II and type B, were purchased from Wako (Wako Pure Chemical Industries, Ltd., Japan).

\section{Untargeted analysis by UPLC-QE-MS Preparation of serum samples}

Blood samples were drawn into Eppendorf tubes and allowed to clot for $30 \mathrm{~min}$ before centrifugation. Then, the samples were centrifuged $\left(1811 \times g, 15 \mathrm{~min}, 4{ }^{\circ} \mathrm{C}\right)$ to obtain serum samples. The serum samples were stored at $-80^{\circ} \mathrm{C}$ until the metabolomics assay. For serum metabolite analysis, all samples were thawed at $4{ }^{\circ} \mathrm{C}$. The serum samples $(100 \mu \mathrm{L})$ and acetonitrile $(400 \mu \mathrm{L})$ were mixed in a tube to remove proteins from the serum, and $\mathrm{L}$, $\mathrm{L}$-dichlorophenylalanine $(60 \mu \mathrm{g} / \mathrm{mL})$ was included as an internal standard. To ensure data quality and instrument stability during metabolic profiling, quality control (QC) samples were prepared by mixing the same volume of serum $(20 \mu \mathrm{L})$ from 78 SHRs and 78 WKYs. The serum samples were shaken in a cryogenic oscillator for $5 \mathrm{~min}$ to mix evenly. Afterwards, the solution was placed at $4{ }^{\circ} \mathrm{C}$ for $15 \mathrm{~min}$, and centrifuged at $15,294 \times g$ for $20 \mathrm{~min}$. The supernatant was transferred into a clean $2 \mathrm{~mL}$ tube, dried with nitrogen and stored at $-20{ }^{\circ} \mathrm{C}$. The samples were resolved with the initial mobile phase prior to LC-MS analysis.

\section{Analysis of serum metabolites}

Ultra performance liquid chromatography-electrospray ionization tandem mass spectrometry (UPLC-ESI-MS/ MS) was performed using an electrostatic field orbitrap mass spectrometer $(\mathrm{QE})$ coupled with an Ultimate 3000 UPLC System (Thermo Fisher, CA, USA). Instrument control, data acquisition and analysis were performed using Xcalibur software 3.0 (Thermo Fisher, CA, USA). The sample vials were maintained at $10{ }^{\circ} \mathrm{C}$ in a thermostatic auto sampler. Chromatographic separation was achieved on a Halo-C18 column $(2.1 \times 100 \mathrm{~mm}, 2.7 \mu \mathrm{m}$;
AMT, USA) with the column temperature set to $45^{\circ} \mathrm{C}$. A total of $5 \mu \mathrm{L}$ of each sample was injected into the column. The mobile phase consisted of solvent A $(0.05 \%$ formic acid in water) and solvent B (0.05\% formic acid in acetonitrile). The linear gradient programme was as follows: 0-1 min, 2\% B; 3 min, 40\% B; 15 min, 98\% B; 17 min, $98 \% \mathrm{~B} ; 17.1 \mathrm{~min}, 2 \% \mathrm{~B}$; and $3.0 \mathrm{~min}$ of equilibration. The flow rate was $0.3 \mathrm{~mL} \cdot \mathrm{min}^{-1}$. Before running the samples, 6 QC samples were run to balance the instrument, and then 1 QC sample was run after every 6 samples. Meanwhile, the mass spectrometry detections were performed as follows: capillary temperature, $350{ }^{\circ} \mathrm{C}$ and spray voltage, $3.5 \mathrm{kV}$ and $3.0 \mathrm{kV}$ for positive ion mode and negative ion mode, respectively. The mass scan range was from 80 to $1200 \mathrm{Da}$. The mass resolution was set to 70,000 .

\section{Multivariate statistical analysis}

The acquired mass spectrometry data (.raw) were exported into Compound Discoverer (CD, Thermo Fisher, CA, USA) software for data analysis. The CD software converts mass spectrometry data into metabolite information. These metabolic discoveries are achieved by using a combination of open online databases, local databases, and MS/MS metabolites data, which greatly improves the accuracy of metabolite identification. The software has the function of multivariate statistical analysis, including the peak area ratio between groups, $\log _{2} \mathrm{FC}$ and significance analysis. To identify the metabolic profiles of the control and model groups, unsupervised principal component analysis (PCA) and supervised partial least-square analysis (PLS-DA) were applied using SIMCA-P version 13.0 (Umetrics AB, Umea, Sweden). The $\mathrm{p}$ value and adjusted $\mathrm{p}$ value for multiple testing of each metabolite were determined by the $C D$ software. Heatmap and pathway analysis were performed with MetaboAnalyst 4.0 software (http://www.metaboanalyst. ca).

\section{Quantitative analysis of amino acids}

Amino acid analysis was performed on a Hitachi L8900 Amino Acid Analyzer with an EZChrom Elite system. Serum samples were thawed at $4{ }^{\circ} \mathrm{C}$. A $100 \mu \mathrm{L}$ serum sample was used for amino acid extraction that used 5-sulfosalicylic acid dehydrate to remove protein. The above sample was diluted with $200 \mu \mathrm{L}$ of $\mathrm{HCl}$ $(0.02 \mathrm{~mol} / \mathrm{L})$ and centrifuged at $15,294 \times g$ for $20 \mathrm{~min}$ before analysis. Chromatographic separation was performed on an ion exchange column. Mobile phases consisted of a buffer solution and ninhydrin. The standard sample was prepared according to the requirements and the amino acids in the serum were quantified by an external standard method. 


\section{Data analysis}

The data are expressed as the mean \pm standard deviation $(\mathrm{SD}, \mathrm{n}=6)$. Statistical significance was determined with SPSS 17.0 software (SPSS Inc., Chicago, IL, USA). Independent sample t-tests were used to assess significant differences. All $\mathrm{p}$ values were two-tailed, and $\mathrm{p}<0.05$ was considered to be significant.

\section{Results}

\section{Baseline description}

A total of 156 rats (78 SHRs and 78WKYs) were included in the study (Fig. 1). General characteristics, including blood pressure, body weight, water intake volume, food intake, face temperature and pain threshold, were shown in Table 1.

To assess the difference in blood pressure and other indicators, the control group and model group were compared by $t$ test. Table 1 shows that the blood pressure of the SHR group was significantly different from that of
Table 1 General characteristics of WKYs and SHRs

\begin{tabular}{lcc}
\hline & Control & Model \\
\hline Body weight $(\mathrm{g})$ & $210 \pm 10$ & $215 \pm 10$ \\
Blood pressure $(\mathrm{mmHg})$ & $111.22 \pm 6.42$ & $181.58 \pm 5.25^{* *}$ \\
Food intake $(\mathrm{g} / \mathrm{d} / \mathrm{cage})$ & $100.06 \pm 11.72$ & $108.78 \pm 13.25$ \\
Volume of drinking water & $192.27 \pm 4.56$ & $167.39 \pm 12.49^{* *}$ \\
$\quad(\mathrm{~mL} / \mathrm{d} /$ cage $)$ & $30.73 \pm 0.29$ & $31.26 \pm 0.72$ \\
Face temperature $\left({ }^{\circ} \mathrm{C}\right)$ & $7.89 \pm 0.74$ & $7.98 \pm 0.25$ \\
Threshold of pain $(\mathrm{S})$ & & \\
\hline
\end{tabular}

${ }^{* *} p<0.01$ the SHR model group compared with the WKY control group

the control group. The body weight was not significantly different. According to the analysis of the volume of water consumed, the rats in the model group consumed much more water than did the rats in the control group $(p<0.01)$. However, the food intake pattern was the

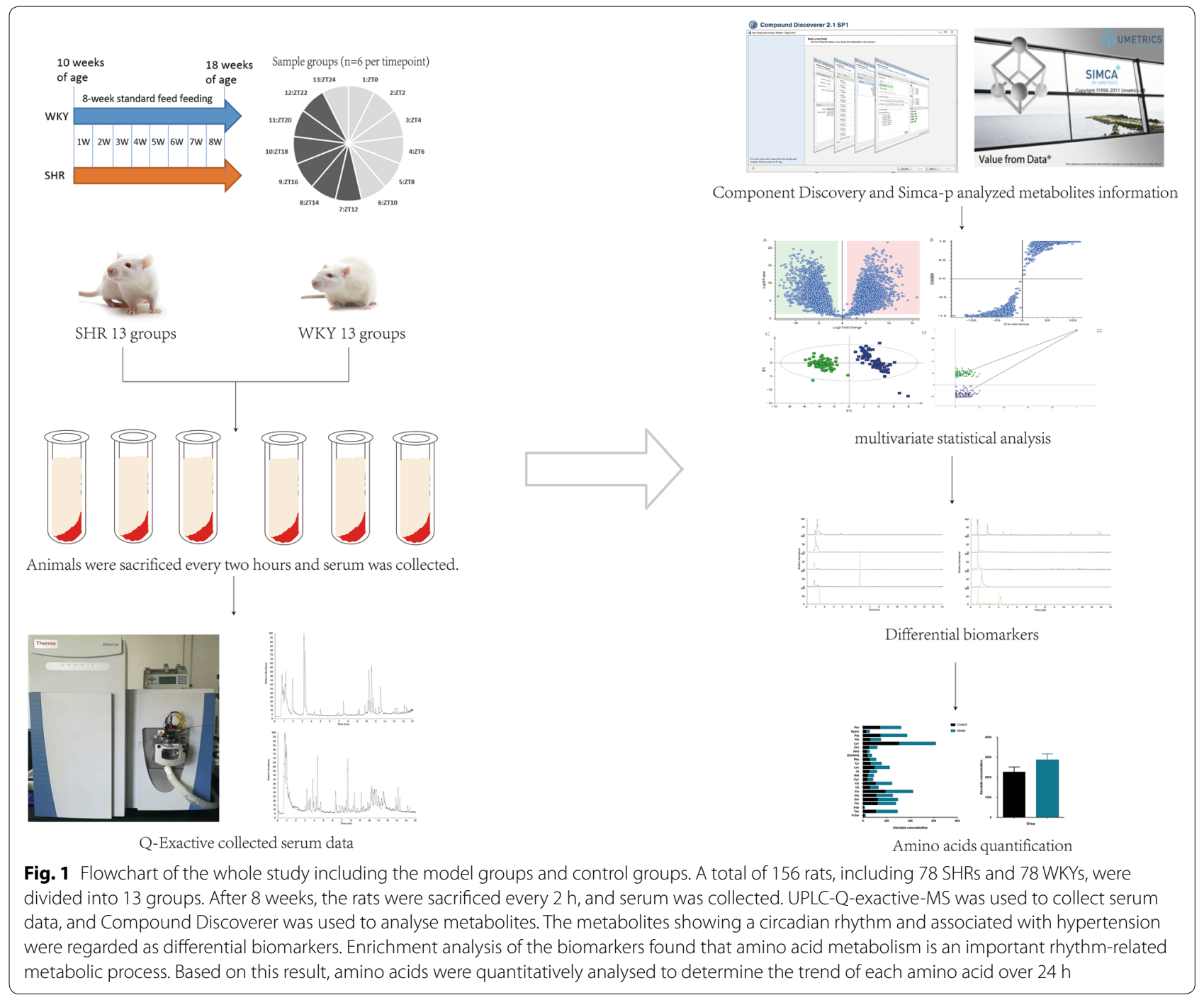


opposite of the water consumption pattern. The differences in the face temperature and pain threshold of the two groups were small and not significant.

\section{Establishment of the circadian metabolic phenotype strategy}

Multivariate statistical analysis was used to assess the daily rhythmicity of the two groups. A total of 112 metabolites showed significant daily rhythms in the control group, and 86 metabolites were significantly different between the control group and the model group $(\mathrm{p}<0.05)$. Among the 112 metabolites, four key metabolites $(20 \%)$ in the tricarboxylic acid cycle (TCA) showed daily rhythms over $24 \mathrm{~h}$, namely, succinic acid, L-malic acid, 2-oxoglutaric acid, and citric acid. Additionally, these metabolites were all included in the 86 differentially expressed metabolites. Ten metabolites $(13 \%)$ in aminoacyl-tRNA biosynthesis pathway (75 metabolites in this pathway) were found in the control group, namely, L-histidine, L-phenylalanine, L-arginine, L-glutamine,
L-valine, L-isoleucine, L-leucine, L-tyrosine, L-proline and L-glutamic acid. Nine metabolites in the arginine and proline metabolism pathways and seven metabolites in the pyrimidine metabolism pathway accounted for $16 \%$ of their metabolic pathways, including L-glutamine, ornithine, citrulline, L-arginine, L-glutamic acid, L-proline, 4-oxoproline, creatine, and creatinine in arginine and proline metabolism, and L-glutamine, uridine, cytidine, cytosine, thymidine, thymine, and pseudouridine in pyrimidine metabolism. This finding indicates that the circadian rhythm is significantly related to the TCA, aminoacyl-tRNA biosynthesis, arginine and proline metabolism, and pyrimidine metabolism. The metabolites with significant differences between the control group and model group were determined by $\log _{2} \mathrm{FC}$ analysis (Fig. 2a), S-plot analysis (Fig. 2b), and PLS-DA (Fig. 2c) [R2Y (cumulative), 0.894; Q2 (cumulative), 0.896]. PLSDA showed a clear separation between the two groups in Fig. 2c. A 100 permutation test (Fig. 2d) was used to verify the accuracy of the model [R2 $=0.939, \mathrm{Q} 2=0.927]$.

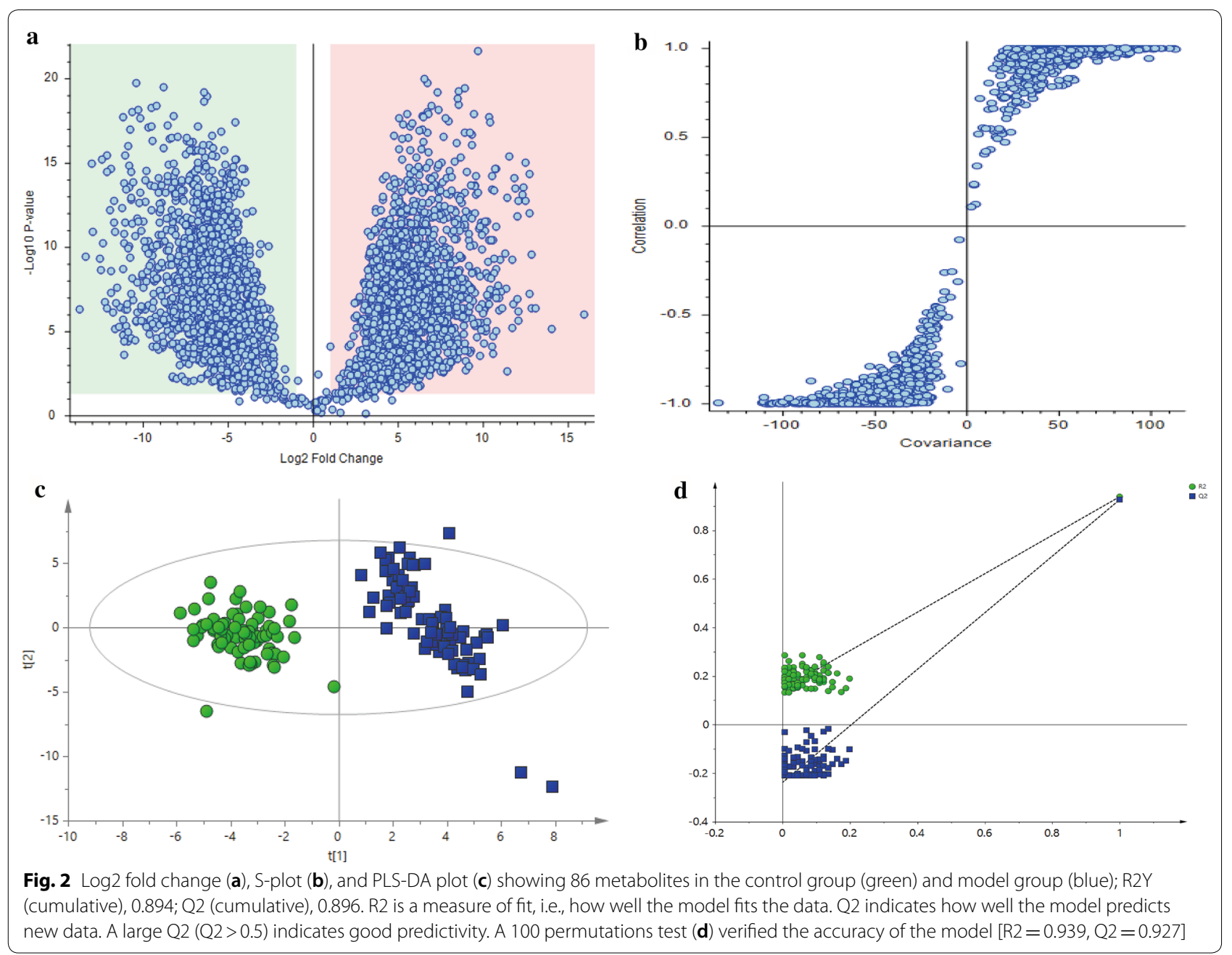


The changes in these metabolites within the model group are shown in the loading plot (Fig. 3a). This plot indicates that mainly amino acids, serotonin, and adrenosterone were positively correlated with the model group and thus, positively correlated with hypertension, whereas glucose, proline, D-tryptophan and citric acid were negatively correlated with hypertension. To maximize the sampling differences between the study groups and to explore the most obvious metabolites with a circadian rhythm, metabolites with a VIP $>1$ were selected (Fig. 3b). The VIP plot was obtained by PLS-DA. The higher the VIP value is, the greater the contribution of that metabolite to the separation of the two groups. Finally, 30 metabolites (36\%) among the 86 metabolites showed significant differences between the two groups, and these metabolites allowed the two groups to separate well in PLS-DA. To further identify biomarkers with significant differences, the metabolic pathways were enriched by analysing markers with a VIP $>1$ (Fig. 3c). The metabolic pathways with $\mathrm{p}<0.05$ were identified as differential metabolic pathways by enrichment analysis. As a result, three differential metabolic pathways were found under these conditions, including the urea cycle, arginine and proline metabolism, and tryptophan metabolism. The biomarkers associated with these differential metabolic pathways included ornithine, glutamine, indoleacetic acid, citrulline, serotonin, L-kynurenine, D-proline, 2-oxoglutaric acid, creatine, and 5-hydroxytryptophan. The expression levels of the abovementioned 10 metabolites in the two groups, as well as the changes over $24 \mathrm{~h}$, are shown by a heatmap of 13 groups $(n=6)$ (Fig. 3d). In the heatmap, red represents the model group and green represents the control group. Moreover, 1 represents 23:00-1:00, 2 represents 1:00-3:00, 12 represents 21:00-23:00, and 13 represents the time blood collection was started, 9:00-11:00. A deep red colour represents a high content, and a dark blue colour represents a low content. Detailed information describing the 10 metabolic biomarkers with rhythmic disorder in the control and model groups is shown in Table 2.

Total ion chromatograms of serum samples were collected by mass spectrometry under the positive and negative ion modes (Fig. 4a: positive; Fig. 4b: negative). The extracted ion chromatogram currents of 10 markers
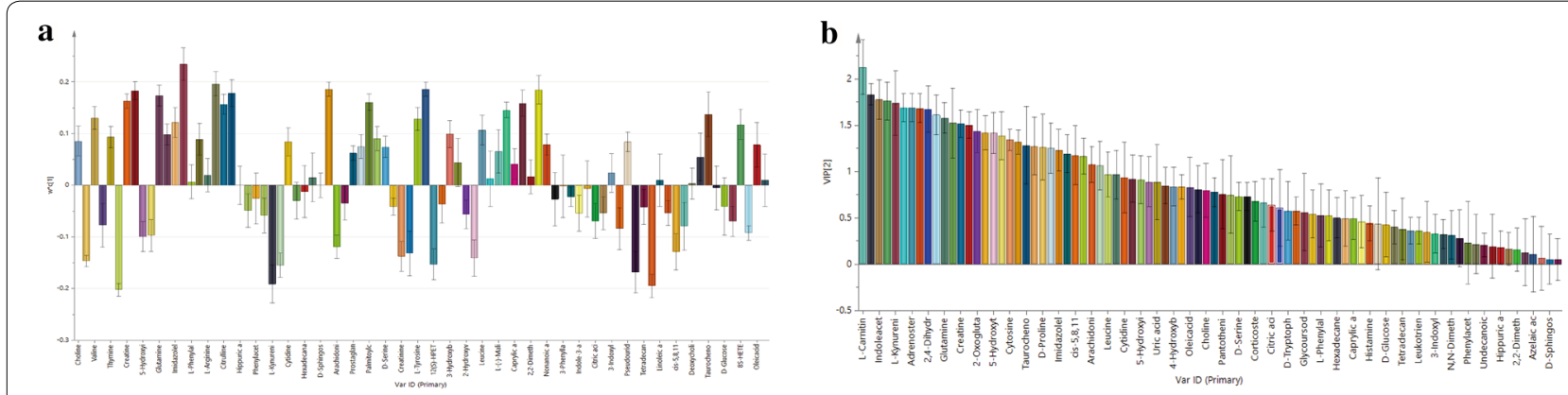

c
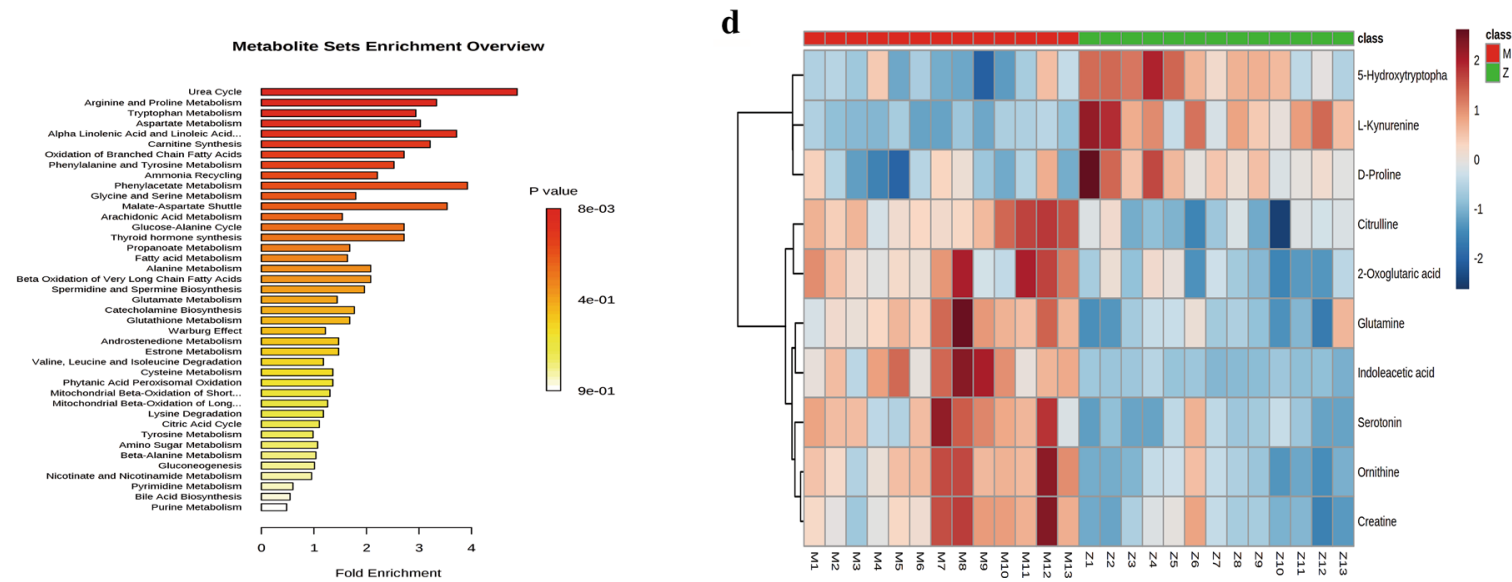

Fig. 3 Loading plot (a) of the model groups and control groups, where the positive values represent metabolites with higher concentrations in the model group. The further away a point is from the $x$-axis, the greater the difference between the two groups. Every two metabolites are labelled on the $x$-axis. VIP plot (b) obtained by PLS-DA. The higher the VIP value is, the greater the contribution to the separation of the two groups. Enrichment analysis (c) of significantly differential metabolites. A higher position and deeper colour demonstrated a strong correlation between the enriched metabolic pathways. Heatmaps (d) of 13 groups $(n=6)$. A deep red colour represent high content, and a dark blue colour represent low content 


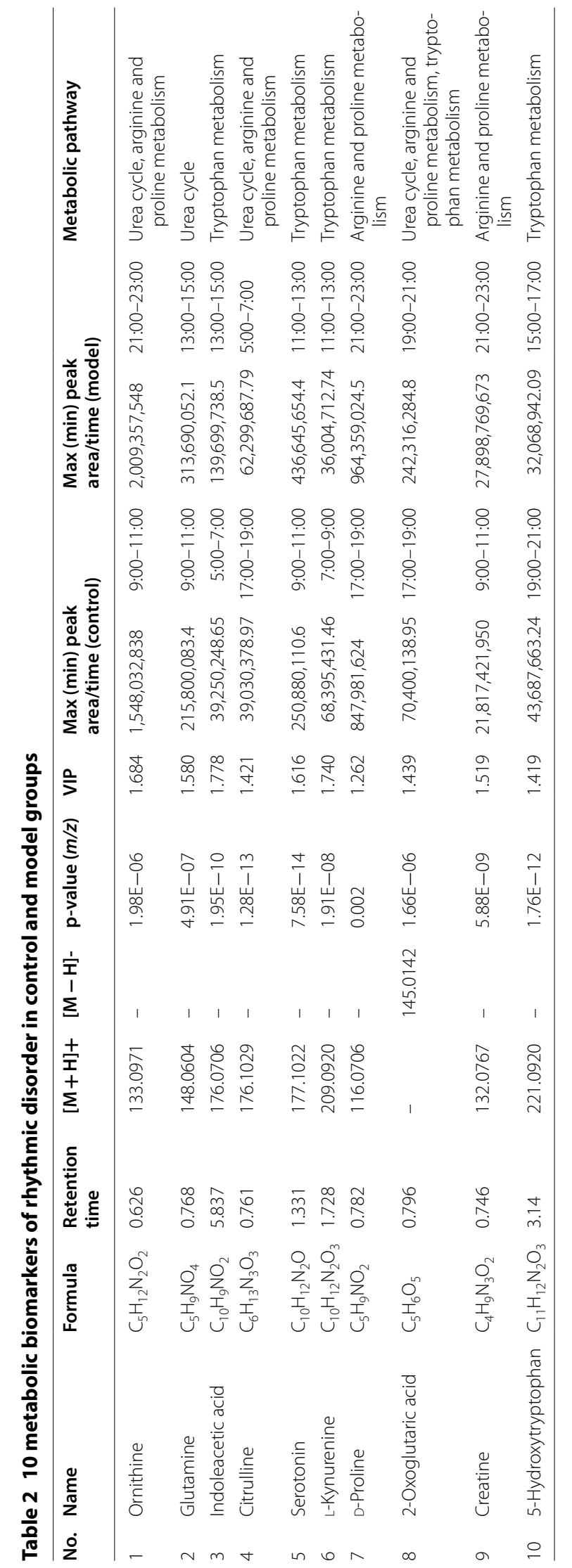


are also shown in Fig. 4c, d. To clearly observe the 24-h change trend of metabolites, the trends of 10 metabolites and their metabolic pathways were mapped, as shown in Fig. 4e, f. The figure shows that the expression patterns of the model group and the control group are different at $24 \mathrm{~h}$, in particular, ornithine, citrulline, creatine and 5-hydroxytryptophan have opposite patterns.

\section{Amino acid and their metabolite contents in serum samples}

Untargeted metabolomics analysis showed that the amino acid and their metabolite contents and the interday variation varied greatly between these two groups. Thus, the amino acids and their metabolites in serum were further quantified by an amino acid analyser. The name, abbreviation, formula, and concentrations of the amino acids and their metabolites detected are shown in Table 3. As shown in Table 3, the number of differential amino acids and their metabolites detected at the six time points over 1 day was different, and 15 amino acids and their metabolites had more than 4 time points differences in $24 \mathrm{~h}$ (Fig. 5a), accounting for $62.5 \%$ of the total number of detected amino acids and their metabolites; At the ZT4, ZT8, and ZT12 time periods, these two groups showed the largest number of differential amino acids and their metabolites (Fig. $5 \mathrm{~b}$ ). Figure $5 \mathrm{c}$ shows the ratio of each amino acid/metabolite to the total amino acids, and Fig. $5 \mathrm{~d}$ shows the absolute concentration of amino acids and their metabolites at ZT8.

\section{Discussion}

Our study used an untargeted metabolomics approach to demonstrate the $24 \mathrm{~h}$ metabolic profile of the SHR group and the WKY control group. SHRs are a well-known model of essential hypertension [15]. An important feature of the SHR model is that it was originally derived from the normal WKY strain [16]. Therefore, in basic scientific research on the level of hypertension animals, WKYs are used as a normal control group for SHRs [2, 17-19]. Metabolomics platforms have been used to measure and identify key biomarkers related to pathological conditions. In recent years, a series of advances have been made in the combined analysis techniques of metabolomics, especially LC-MS and nuclear magnetic resonance (NMR) [20, 21]. Circadian rhythm research also relies on these platforms, as metabolomics can reflect the whole physiological state by the detection of specific metabolites [6, 9, 14]. These high-throughput, high-sensitivity and high-resolution research platforms have promoted a series of studies on circadian rhythm metabolism.

In this study, the 24-h rhythms of serum metabolites in rats were characterized by using LC-MS metabolomics, including arginine, proline, serotonin, phenylalanine, citric acid, L-malic acid and succinic acid, etc., offering novel insight into hypertension. In addition, based on the untargeted metabolomics outcomes the concentrations of amino acids and their metabolites were determined, and the changes in leucine, isoleucine, valine, taurine, serine, and glycine were the most obvious. In the current hypertension-circadian rhythm study, the metabolic profile of SHRs was found to be different from that of WKYs from a holistic perspective. The mass spectral intensity of 112 metabolites varied at different time points, indicating that the concentration of metabolites constantly changes throughout the day. Multivariate statistical analysis was used to identify differential metabolites between the control and model groups and rhythmic biomarkers. The results from this study suggested that the circadian rhythm of hypertension is closely related to the urea cycle, arginine and proline metabolism and tryptophan metabolism. Interestingly, the disruption of metabolic pathways that occurs in many metabolic disorders has been reported to impact gene expression programmes in tissues and organ physiology by affecting molecular clock rhythms [22, 23].

The metabolic pathways that link hypertension with circadian rhythm are not well understood. Metabolic pathway enrichment analysis revealed that the urea cycle, which includes ornithine, purine, glutamic acid, citrulline and 2-oxoglutaric acid, was the most significantly impacted metabolic pathway. Notably, the urea cycle also known as the ornithine cycle, is the final pathway in the removal of surplus nitrogen from the body, as well as the major route for the detoxification of ammonia [24]. As energy is required to dispose of excess ammonia, it is apparent that ATP must be available for the urea cycle to properly function. Thus, the urea cycle is closely linked to the citric acid cycle, which derives one of its nitrogens from the transamination of oxaloacetate to form aspartate and returns fumarate to the cycle [25, 26].It is reported that an LC-MS method was used to quantify amino acids to investigate urea cycle disorders. It discovered that urea cycle disorder not only disturbed the metabolism of amino acids involved in the urea cycle and induced the accumulation of ammonia detoxification, but also interfered with the metabolism of amino acids related to some nervous systems, such as pipecolic acid and N-acetylaspartic acid [27]. Our research was consistent with this study. During the development of hypertension, the contents of amino acids such as ornithine, glutamic acid and citrulline involved in the urea cycle were higher in the hypertension group than in the control group, and the urea cycle reaction substrates were abundant, resulting in an increase in blood ammonia. In addition, arginase, the enzyme that converts arginine 

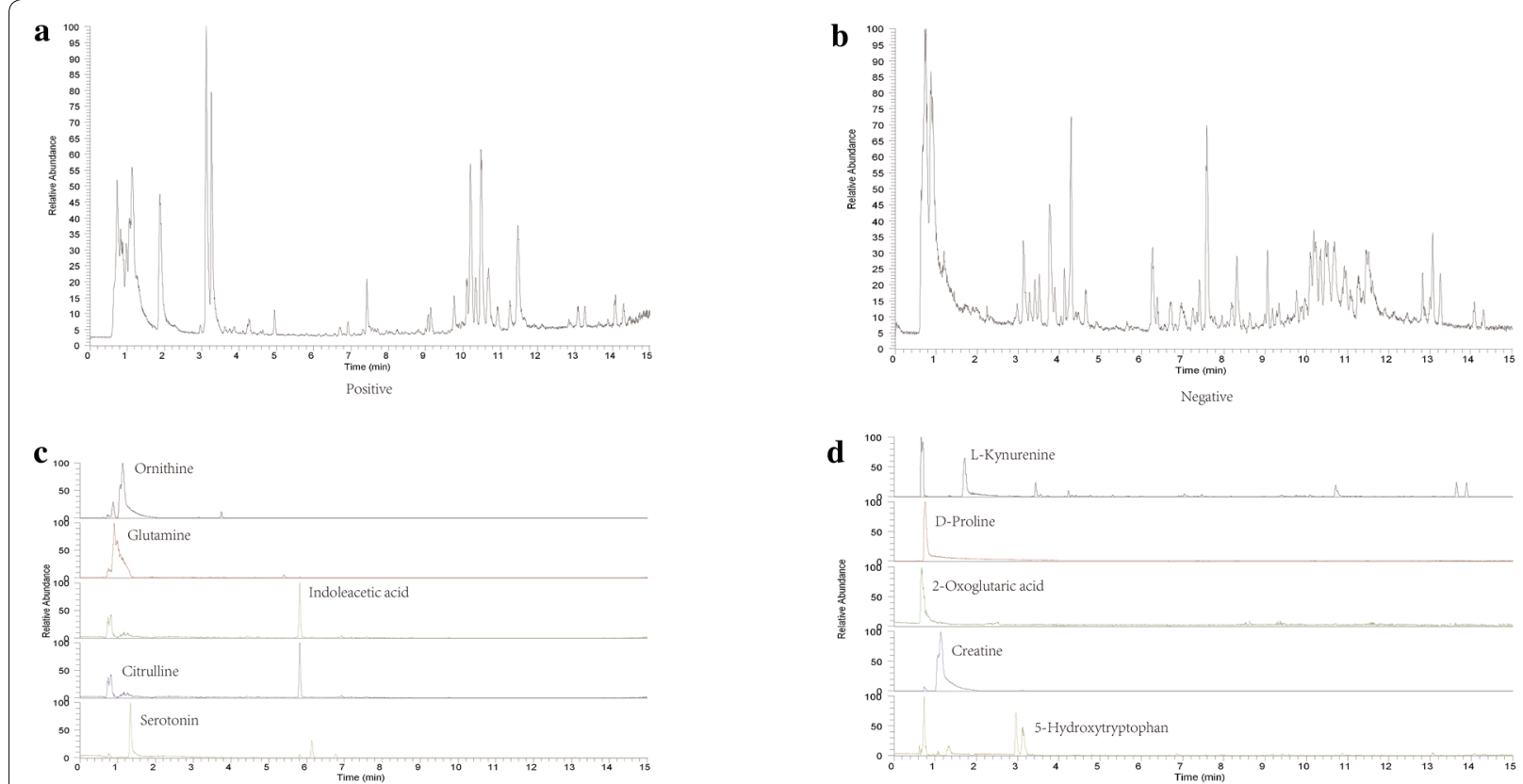

e

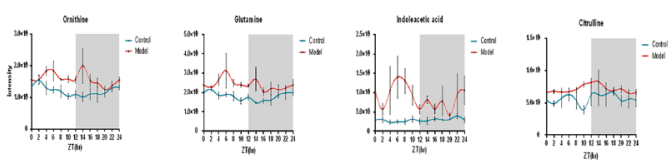

\section{f}
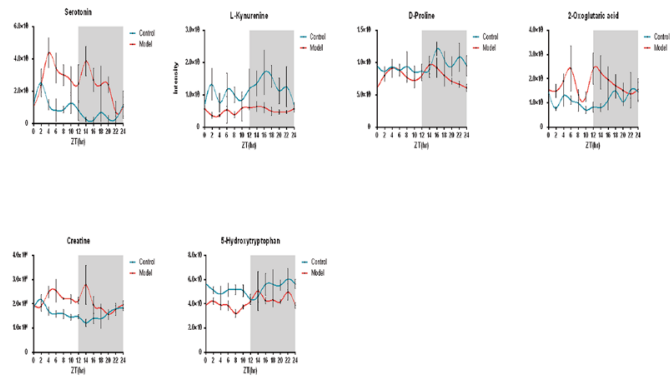

Fig. 4 Total ion chromatogram of serum was collected by mass spectrometry under positive and negative ion modes (a: positive; b: negative). Amino acids were mostly collected in the positive ion mode. c, $\mathbf{d}$ The extracted ion currents of 10 markers, and most substances peaked within 5 min. e, f The 24-h trend charts of 10 metabolites and metabolic pathways. The amplitude and expression of the model group and the control group are different at $24 \mathrm{~h}$, in which ornithine, citrulline, creatine and 5-hydroxytryptophan have opposite trends. Zeitgeber time (ZT) 0 represents lights on. The metabolic pathways included glycolysis, urea metabolism, the TCA, tyrosine metabolism, and tryptophan metabolism

to ornithine and urea, competes with oxide synthetase (NOS) for arginine leading to a decrease in nitric oxide (NO) expression and the destruction of the urea cycle. As $\mathrm{NO}$ is a critical factor in cell growth and vasodilation, and the decrease of in NO leads to increased hypertension $[28,29]$. The 24-h metabolite rhythms in overweight/ obese individuals and in individuals with type 2 diabetes showed that proline has robust daily rhythms and that isoleucine and valine rhythms were disturbed in patients with type 2 diabetes $[6,9]$. Our animal experiments also found similar results. The serum content of branchedchain amino acids (BCAAs) was significantly higher in the hypertension groups compared with the control groups, which indicated that animal experiments could reflect the changes in the human body to a certain extent. BCAAs may affect cell signalling and aggravate oxidative 


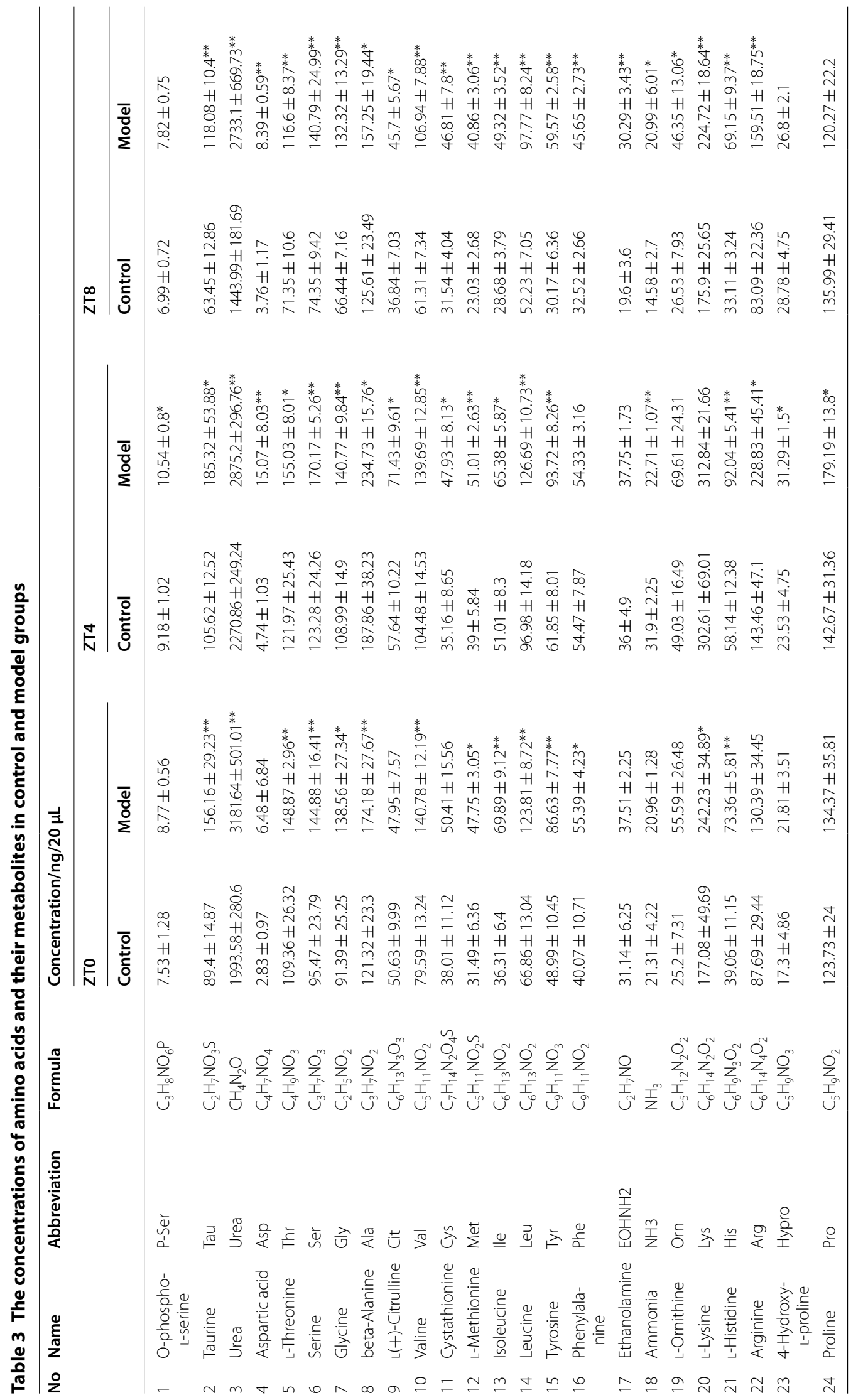




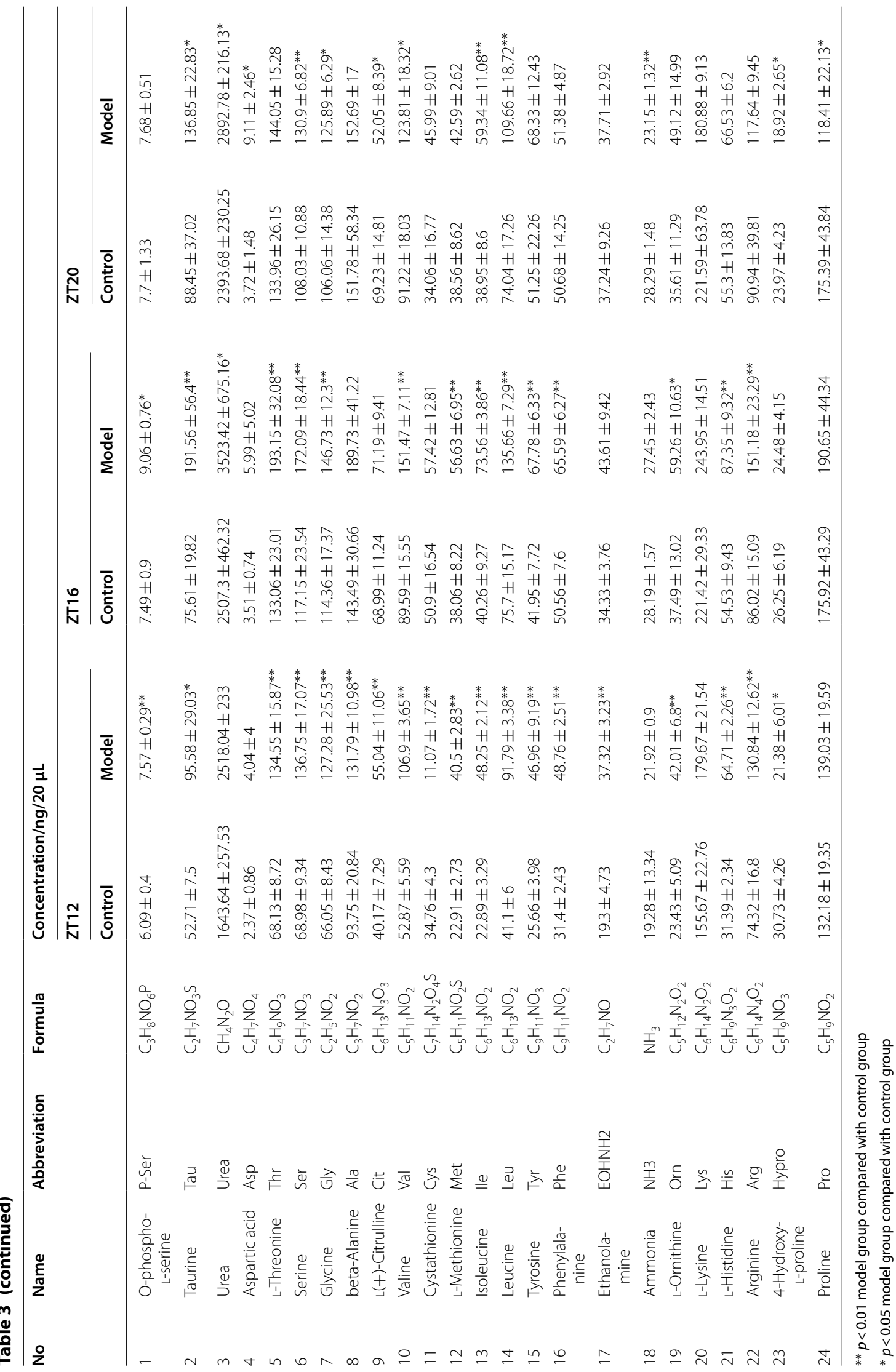




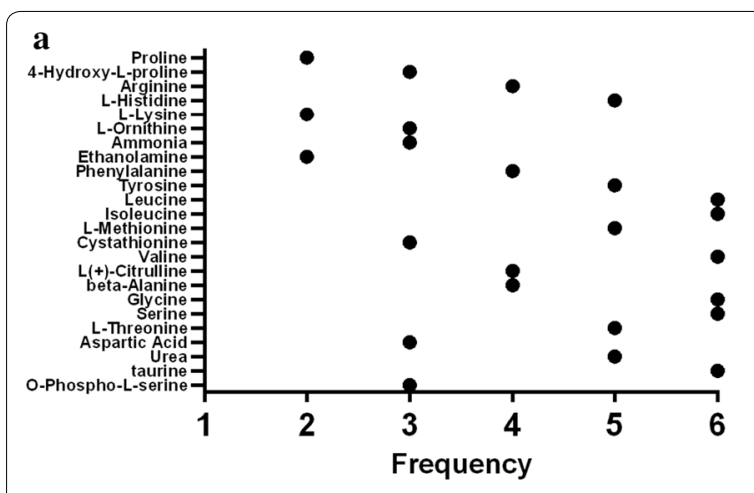

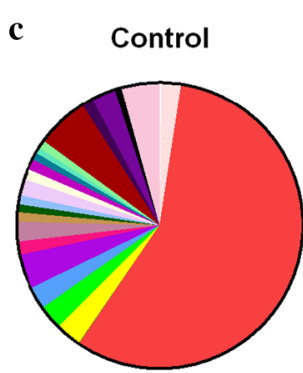

Total $=3379.3$ $\square 0.22 \%$ P-Ser

$\square 2.21 \%$ Tau

$\square 57.06 \%$ Urea

$\square 0.10 \%$ Asp

$\square 2.91 \% \mathrm{Thr}$

$\square 2.73 \%$ Ser

$\square 2.56 \%$ Gly

$\square 3.91 \%$ Ala

$\square 1.49 \%$ Cit

$\square 2.22 \%$ Val

$\square 1.08 \%$ Cys

0 $0.89 \%$ Met $\square 1.01 \%$ Ile

$\square 1.88 \%$ Leu

$\square 1.18 \% \mathrm{Tyr}$

$\square 1.21 \%$ Phe

$\square 0.81 \%$ EOHNH2

$\square 0.66 \% \mathrm{NH} 3$

$\square 0.93 \%$ Orn

$\square .94 \%$ Lys

च $1.26 \% \mathrm{His}$

$\square 2.70 \%$ Arg

- $0.78 \%$ Hypro

$\square 4.30 \%$ Pro

b
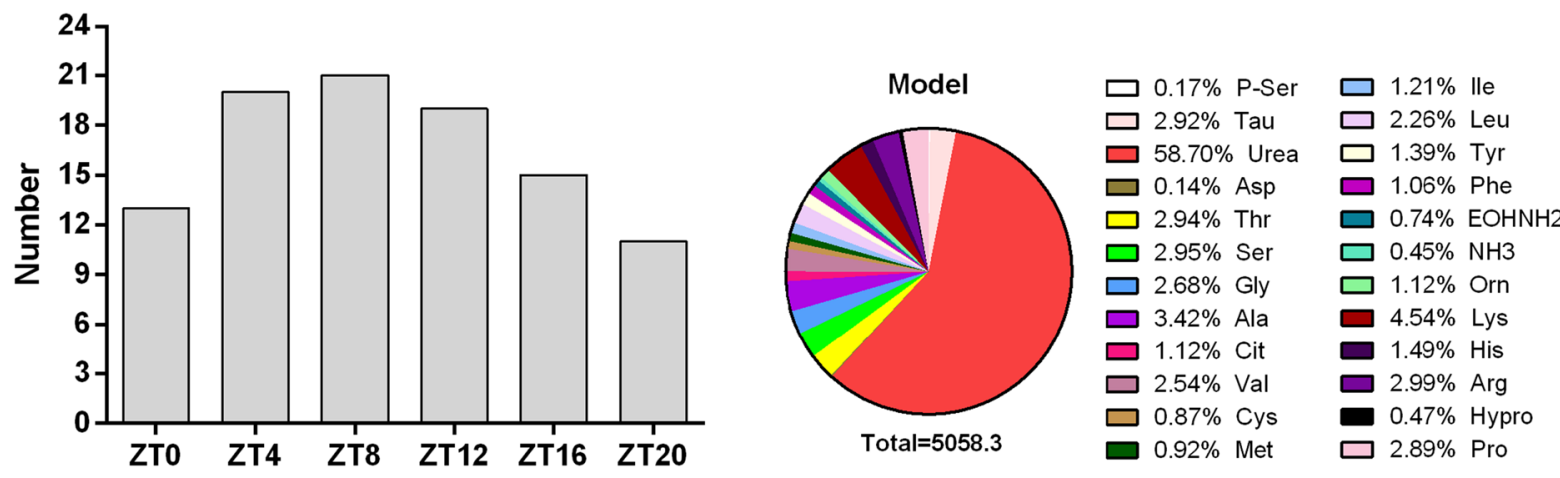

d

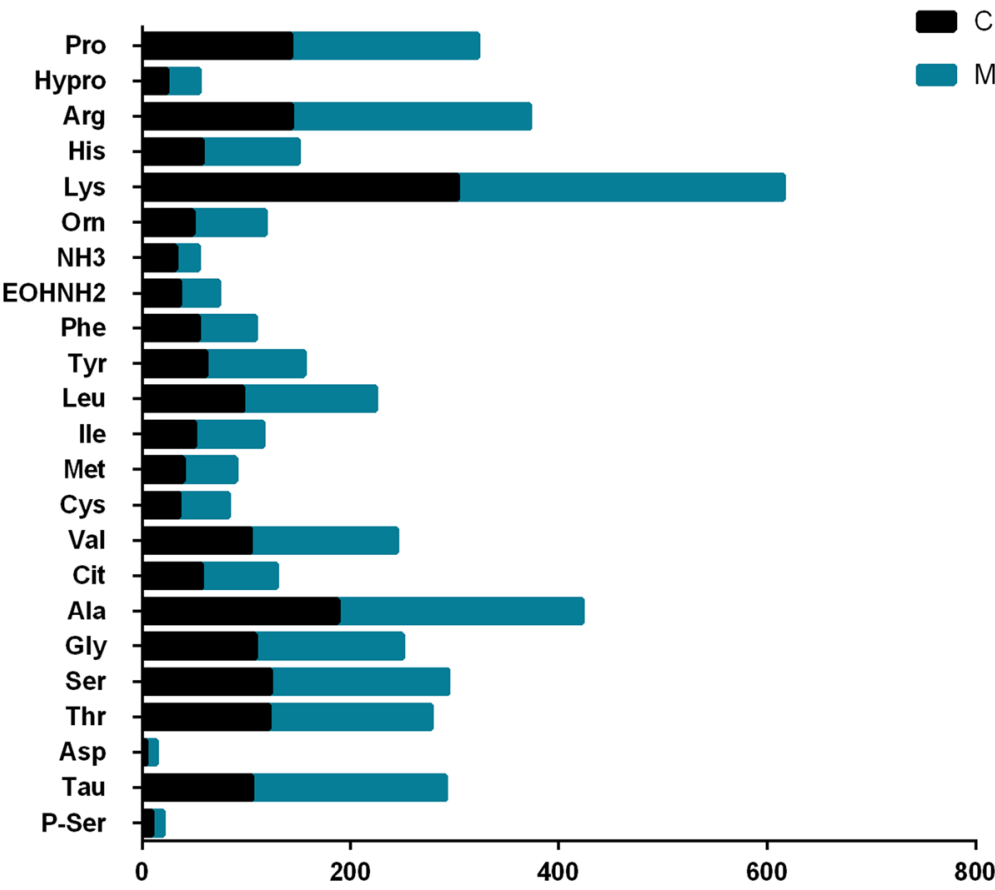

Control

Model
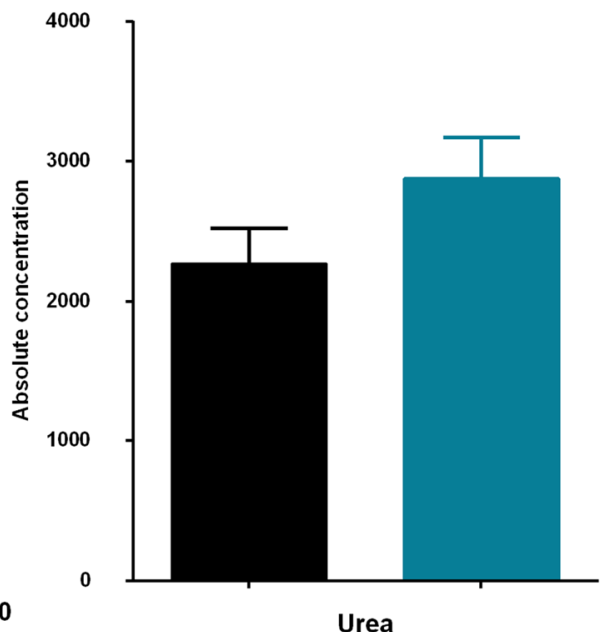

Absolute concentration

Fig. 5 a The frequency of differences in the amino acids and their metabolites detected at 6 time points in a day, and $\mathbf{b}$ the number of amino acids that differed at the different detection times during the day. $\mathbf{c}$ The ratio of each amino acid/metabolite to the total amino acids and their metabolites in the serum. Different amino acids and their metabolites are identified by different colours. Higher total amino acid content in the serum, higher urea content in the serum, and lower aspartic acid content was observed in the model group than in the control group. $\mathbf{d}$ The absolute concentration of amino acids and their metabolites at ZT8 
stress [30], which contributes to endothelial cell damage. Endothelial cell damage is one of the causative factors of hypertension [31]. Therefore, in addition to cell signalling and oxidative stress, high concentrations of BCAAs are commonly associated with hypertension. These results also indicated the role of the circadian metabolic processes in hypertension.

Our pathway analysis revealed that arginine and proline metabolism were enriched in hypertension. Both in vivo and in vitro experiments have demonstrated that amino acids are circadian [32]. In general, amino acids rarely remain free in cells, because they have many physiological functions, such as (i) protein synthesis in a fed state; (ii) gluconeogenesis during fasting; (iii) metabolism into bioactive molecules; or (iv) degradation to liberate ammonia, which is fed into the urea cycle [33]. In a randomized, double-blind, crossover trial investigating air purification, 10 amino acids (arginine, leucine, histamine, threonine, serine, glutamine, lysine, phenylalanine, tyrosine, and tryptophan) were also clearly associated with PM2.5, which has been proven to be related to oxidative stress, which is associated with elevated blood pressure [34]. L-Histidine, L-phenylalanine, L-arginine, L-glutamine, L-valine, L-isoleucine, L-leucine, L-tyrosine, L-proline, and L-glutamic acid showed rhythm disturbances in hypertension based on the metabolomics method. In addition to amino acids, metabolites in the TCA such as succinic acid, L-malic acid, 2-oxoglutaric acid, and citric acid, and glucose also had abnormal rhythms and contents in hypertension. These results showed that amino acids may affect energy metabolism by increasing metabolic rate and energy expenditure to meet the physical needs in hypertensive patients with TCA inhibition. Moreover, an increase in arginine and proline metabolism is closely related to arterial injury. As mentioned above, arginase and NOS competitively bind to arginine to produce $\mathrm{NO}$ and ornithine (the precursor of polyamines and proline), respectively [35]. An increase in polyamine synthesis has been observed in response to the proliferation of vascular smooth muscle cells and the development of intimal thickening [36], which could also contribute to hypertension. Moreover, it has been hypothesized that amino acids change significantly in the circadian misalignment individuals with of type 2 diabetes, prediabetes/obesity, reduced insulin sensitivity and impaired glucose homeostasis and those consuming a high-fat diet [37, 38]. This hypothesis was also confirmed in our study on hypertension rhythm, indicating that changes in amino acid contents are an independent risk factor for cardiovascular disease.

It has been reported that amino acids in human plasma, including tryptophan, tyrosine, phenylalanine, methionine, cysteine, glutathione and homocysteine have circadian rhythms [39-42]. Because amino acids can be used for gluconeogenesis under energy deprivation [43], rhythm disorders in the catabolism of amino acids, especially BCAAs, including leucine, isoleucine, and valine, can lead to serious cardiovascular diseases [44]. Serum amino acids are markers of prediabetes, insulin resistance and incident diabetes [45]. Abnormal amino acid metabolism was also observed in hypertension. The quantitative analysis of amino acids revealed that at least one amino acid was significantly different at the same time and each amino acid had at least one time difference within a day. Circadian clocks are fundamental physiological regulators of energy homeostasis. For instance, the loss of bmal1, a circadian clock gene, is associated with the accumulation of amino acids [46]. Accordingly, amino acid contents can reflect the expression of circadian clock genes. In this study, the accumulation of BCAAs was found by amino acid quantification, which revealed that hypertension causes metabolic deficiencies in BCAAs. Additionally, serum amino acids were consistently higher in the hypertension group than in the control group over $24 \mathrm{~h}$. High concentrations of BCAAs promote oxidative stress, inflammation and migration via mTORC1 activation [47]. In addition, leucine, a branched-chain amino acid, can activate mTORC1 signalling to increase sympathetic nerve activity and arterial pressure in anaesthetized rats [48-50]. Therefore, it is speculated that BCAAs are also mainly related to elevated blood pressure through the mTORC1 pathway.

Tryptophan metabolism also showed an oscillatory imbalance in hypertension except for the urea cycle and arginine, proline and BCAA metabolism. In the tryptophan metabolism pathway, the concentrations of indoleacetic acid, serotonin, and 2-oxoglutaric acid increased, while the concentrations of L-kynurenine and 5-hydroxytryptophan decreased. Interestingly, kynurenine, a marker of renal function, was also altered by prior exposure to simulated night-shift work $[6,51]$. It has been reported that kynurenine serves as a negative feedback regulator in the event of increased vascular pressure. In arteries, kynurenine may cause arterial relaxation mainly via the cAMP pathway, ameliorating the constantly increased wall stress [52]. However, a continuous increase in arterial blood pressure and a decrease in kynurenine expression indicate that the steady state of tryptophan metabolism is destroyed and the feedback loop is out of order, which in turn leads to an increase in blood pressure. Hypertension patients may have suppressed secretion of melatonin, a hormone that regulates the circadian cycle and has multiple cardioprotective properties [53]. Additionally, melatonin has been discovered to have antihypertensive effects, most likely because of its anti-inflammatory and reactive oxygen species 
scavenging properties [54]. 5-Hydroxytryptophan produces serotonin under the action of tryptophan decarboxylase. Through additional reactions, serotonin can produce $\mathrm{N}$-acetylserotonin, the precursor of melatonin under the action of serotonin acetylase [34]. Our study showed that serotonin levels increase but that melatonin levels decrease in hypertension. This finding may indicate that changes in the content or activity of enzymes occur during the production of downstream metabolites of serotonin, which may be helpful to elucidate the pathogenesis of hypertension.

Compared with SHRs, human hypertension developed with many risk factors, including ethnicity, familial, dyslipidemia, and lifestyle [55]. Base on this, hypertension patients generally accompany with other co-morbidities, such as obesity, diabetes, left ventricular hypertrophy, albuminuria and renal dysfunction [56]. Although there may be some differences in the results of the experiments translating into humans, the pathogenesis of human hypertension is approximately similar to that of SHRs. The common mechanism of vascular injury in hypertension patients and SHRs including endothelial dysfunction, atherosclerosisi, and is associated with an imbalance of vascular NO bioavailability and oxidative stress [57]. Moreover, compared with hypertension patients, the metabolic pathways of SHRs to some extent show similarities. For instance, in the study of metabolomics on human hypertension, it found that the differential biomarkers are closely related to amino acid metabolism [58], and the study of young hypertensive men on metabolomics also indicate that variances of amino acids have a major impact on the metabolic change [59]. In addition, metabolites variability within a $24 \mathrm{~h}$ period has been closely associated with metabolic disorders $[6,9]$. Therefore, the circadian metabolic phenotype strategy of our study would be translated and explained the mechanism of metabolic rhythm of hypertension patients.

Our study also has several limitations. The central circadian clock genes and the peripheral circadian clock genes were not detected, so the relationship between metabolites and genes was not elucidated. However, it has been reported that the loss of the endogenous circadian clock can lead to the destruction of metabolic homeostasis [60]. Temporal profiles may be controlled by the SCN clock [61]. Although, it is currently understood that the circadian rhythm is regulated by negative feedback loops to ensure that cells, organisms and metabolites are consistent with the light-dark cycle, important questions remain unknown regarding the biophysical mechanisms that underlie transcriptional oscillations and the regulation of metabolites $[62,63]$.

\section{Conclusion}

The mechanism of hypertension in general physiology and pathology has been widely studied, and a large amount of knowledge has been gathered in the study of antihypertensive drugs. Nevertheless, the effect of circadian rhythm on metabolites is also not negligible. Under the guidance of chronobiology and timing-based administration, the study of the dynamics of hypertension is particularly important for the interpretation of the mechanism. From this study, metabolite concentrations constantly change throughout the day, and the amplitude and phase of these changes that occur in hypertension deviate. These rhythmic indicators should also be included in future research and treatment. In this study, the results not only showed that the metabolic profiles of SHRs were different from those of the control group, but also revealed that the endogenous metabolites in SHRs have different characteristics. In particular, the 24-hour oscillation patterns of ornithine, citrulline, creatine and 5-hydroxytryptophan in the hypertension group were different from those in the control group, and BCAAs accumulated in hypertension. These findings provide good further research directions and may help to reveal the pathology of hypertension.

\begin{abstract}
Abbreviations
TCA: tricarboxylic acid cycle; SCN: suprachiasmatic nucleus; LC-MS: liquid chromatography-mass spectrometry; NMR: nuclear magnetic resonance; SHRs: spontaneously hypertensive rats; WKY: Wistar-Kyoto Rats; QC: quality control; CD: compound Discoverer; PCA: principal component analysis; PLSDA: partial least-square analysis; BCAAs: branched-chain amino acids; NOS: nitric oxide synthase; NO: nitric oxide.
\end{abstract}

\section{Acknowledgements}

Not applicable.

\section{Authors' contributions}

HW, XW conducted LC-MS analysis and draft the manuscript. DQ conducted data analysis. MS, QH conducted animal feeding and handing. YL and $\mathrm{HJ}$ were research conductor. All authors read and approved the final manuscript.

\section{Funding}

This work was supported by foundation from the National Natural Science Foundation of China (81774173), Shandong Province major scientific and technological innovation (2017CXGC1307), Shandong Province key research and development plan (2018GSF119007), and Major Basic Research Projects of Shandong Natural Science Foundation (ZR2018ZC1157).

Availability of data and materials

Not applicable.

Ethics approval and consent to participate

All animal studies were approved by the Animal Ethics Committee of Shandong University of Traditional Chinese Medicine.

\section{Consent for publication \\ Not applicable.}

\section{Competing interests}

The authors declare that they have no competing interests. 


\section{Author details}

${ }^{1}$ School of Pharmaceutical Sciences, Shandong University of Traditional Chinese Medicine, Jinan 250355, People's Republic of China. ${ }^{2}$ Experimental Center, Shandong University of Traditional Chinese Medicine, Jinan 250355, People's Republic of China. ${ }^{3}$ Key Laboratory of Traditional Chinese Medicine Classical Theory, Ministry of Education, Shandong University of Traditional Chinese Medicine, Jinan 250355, People's Republic of China. ${ }^{4}$ Shandong Provincial Key Laboratory of Traditional Chinese Medicine for Basic Research, Shandong University of Traditional Chinese Medicine, Jinan 250355, People's Republic of China. ${ }^{5}$ TCM Clinical Research Base for Hypertension, Affiliated Hospital of Shandong University of Traditional Chinese Medicine, Jinan 250011, People's Republic of China.

\section{Received: 10 September 2019 Accepted: 10 January 2020} Published online: 28 January 2020

\section{References}

1. O'Shea PM, Griffin TP, Fitzgibbon M. Hypertension: the role of biochemistry in the diagnosis and management. Clin Chim Acta. 2017:465:131-43.

2. Tian Y, Jiang F, Li Y, Jiang H, Chu Y, Zhu L, et al. Evaluation of the anti-hypertensive effect of Tengfu Jiangya tablet by combination of UPLC-Q-exactive-MS-based metabolomics and iTRAQ-based proteomics technology. Biomed Pharmacother. 2018;100:324-34.

3. Liu A, Chu YJ, Wang X, Yu R, Jiang H, Li Y, et al. Serum metabolomics study based on Ic-ms and antihypertensive effect of uncaria on spontaneously hypertensive rats. Evid Based Complement Alternat Med. 2018;2018:9281946.

4. Li Y, Li G, Gorling B, Luy B, Du J, Yan J. Integrative analysis of circadian transcriptome and metabolic network reveals the role of de novo purine synthesis in circadian control of cell cycle. PLoS Comput Biol. 2015;11:e1004086.

5. Brown SA. Circadian metabolism: from mechanisms to metabolomics and medicine. Trends Endocrinol Metab. 2016;27:415-26.

6. Skene DJ, Skornyakov E, Chowdhury NR, Gajula RP, Middleton B, Satterfield BC, et al. Separation of circadian- and behavior-driven metabolite rhythms in humans provides a window on peripheral oscillators and metabolism. Proc Natl Acad Sci USA. 2018;115:7825-30.

7. Minami Y, Kasukawa T, Kakazu Y, ligo M, Sugimoto M, Ikeda S, et al. Measurement of internal body time by blood metabolomics. Proc Natl Acad Sci. 2009;106:9890-5.

8. Abbondante S, Eckel-Mahan KL, Ceglia NJ, Baldi P, Sassone-Corsi P. Com parative circadian metabolomics reveal differential effects of nutritional challenge in the serum and liver. J Biol Chem. 2016;291:2812-28.

9. Isherwood CM, Van der Veen DR, Johnston JD, Skene DJ. Twenty-fourhour rhythmicity of circulating metabolites: effect of body mass and type 2 diabetes. FASEB J. 2017;31:5557-67.

10. Gooley JJ. Circadian regulation of lipid metabolism. Proc Nutr Soc. 2016;75:440-50.

11. Antunes LC, Levandovski R, Dantas G, Caumo W, Hidalgo MP. Obesity and shift work: chronobiological aspects. Nutr Res Rev. 2010;23:155-68.

12. Kecklund $G$, Axelsson J. Health consequences of shift work and insufficient sleep. BMJ. 2016;355:15210.

13. Altman BJ, Hsieh AL, Sengupta A, Krishnanaiah SY, Stine ZE, Walton ZE, et al. MYC disrupts the circadian clock and metabolism in cancer cells. Cell Metab. 2015;22:1009-19.

14. Dyar KA, Lutter D, Artati A, Ceglia NJ, Liu Y, Armenta D, et al. Atlas of circadian metabolism reveals system-wide coordination and communication between clocks. Cell. 2018;174(1571-85):e11.

15. Yeves AM, Burgos Jl, Medina AJ, Villa-Abrille MC, Ennis IL. Cardioprotective role of IGF-1 in the hypertrophied myocardium of the spontaneously hypertensive rats: a key effect on NHE-1 activity. Acta Physiol (Oxf). 2018;224:e13092.

16. Gauthier AC, DeAngeli NE, Bucci DJ. Cross-fostering differentially affects ADHD-related behaviors in spontaneously hypertensive rats. Dev Psychobiol. 2015;57:226-36.

17. le Noble JL, Smith TL, Hutchins PM, Struyker-Boudier HA. Microvascular alterations in adult conscious spontaneously hypertensive rats. Hypertension. 1990;15:415-9.
18. Fenoy FJ, Kauker ML, Milicic I, Roman RJ. Normalization of pressure-natriuresis by nisoldipine in spontaneously hypertensive rats. Hypertension. 1992;19:49-55

19. Wilson C, Zhang X, Buckley C, Heathcote HR, Lee MD, McCarron JG. Increased vascular contractility in hypertension results from impaired endothelial calcium signaling. Hypertension. 2019;74:1200-14.

20. Ribas-Latre A, Del Bas JM, Baselga-Escudero L, Casanova E, Arola-Arnal A, Salvado MJ, et al. Dietary proanthocyanidins modulate melatonin levels in plasma and the expression pattern of clock genes in the hypothalamus of rats. Mol Nutr Food Res. 2015;59:865-78.

21. Zhao L, Dong M, Ren M, Li C, Zheng H, Gao H. Metabolomic analysis identifies lactate as an important pathogenic factor in diabetes-associated cognitive decline rats. Mol Cell Proteomics. 2018;17:2335-46.

22. Turek FW, Joshu C, Kohsaka A, Lin E, Ivanova G, McDearmon E, et al. Obesity and metabolic syndrome in circadian Clock mutant mice. Science. 2005;308:1043-5.

23. Rey G, Valekunja Utham K, Feeney Kevin A, Wulund L, Milev Nikolay B, Stangherlin A, et al. The pentose phosphate pathway regulates the circadian clock. Cell Metab. 2016;24:462-73.

24. Walker $\mathrm{V}$. Ammonia toxicity and its prevention in inherited defects of the urea cycle. Diabetes Obes Metab. 2009;11:823-35.

25. Salway JG. The krebs uric acid cycle: a forgotten krebs cycle. Trends Biochem Sci. 2018;43:847-9.

26. Shambaugh GE 3rd. Urea biosynthesis I The urea cycle and relationships to the citric acid cycle. Am J Clin Nutr. 1977;30:2083-7.

27. Peng MZ, Cai YN, Shao YX, Zhao L, Jiang MY, Lin YT, et al. Simultaneous quantification of 48 plasma amino acids by liquid chromatographytandem mass spectrometry to investigate urea cycle disorders. Clin Chim Acta. 2019;495:406-16.

28. Nagamani SC, Erez A, Lee B. Argininosuccinate lyase deficiency. Genet Med. 2012;14:501-7.

29. Zheng HK, Zhao JH, Yan Y, Lian TY, Ye J, Wang XJ, et al. Metabolic reprogramming of the urea cycle pathway in experimental pulmonary arterial hypertension rats induced by monocrotaline. Respir Res. 2018;19:94.

30. Flores-Guerrero JL, Groothof D, Connelly MA, Otvos JD, Bakker SJL, Dullaart RPF. Concentration of branched-chain amino acids is a strong risk marker for incident hypertension. Hypertension. 2019;74(6):1428-35.

31. Incalza MA, D'Oria R, Natalicchio A, Perrini S, Laviola L, Giorgino F. Oxidative stress and reactive oxygen species in endothelial dysfunction associated with cardiovascular and metabolic diseases. Vascul Pharmacol. 2018;100:1-19.

32. Krishnaiah SY, Wu G, Altman BJ, Growe J, Rhoades SD, Coldren F, et al. Clock regulation of metabolites reveals coupling between transcription and metabolism. Cell Metab. 2017;25:961-74.

33. Panda S. Circadian physiology of metabolism. Science. 2016;354:1008-15.

34. Li H, Cai J, Chen R, Zhao Z, Ying Z, Wang L, et al. Particulate matter exposure and stress hormone levels: a randomized, double-blind, crossover trial of air purification. Circulation. 2017;136:618-27.

35. Getz GS, Reardon CA. Arginine/arginase NO NO NO. Arterioscler Thromb Vasc Biol. 2006;26:237-9.

36. Martin-Lorenzo M, Zubiri I, Maroto AS, Gonzalez-Calero L, Posada-Ayala $\mathrm{M}$, de la Cuesta F, et al. KLK1 and ZG16B proteins and arginine-proline metabolism identified as novel targets to monitor atherosclerosis, acute coronary syndrome and recovery. Metabolomics. 2015;11:1056-67.

37. Newgard CB, An J, Bain JR, Muehlbauer MJ, Stevens RD, Lien LF, et al. A branched-chain amino acid-related metabolic signature that differentiates obese and lean humans and contributes to insulin resistance. Cell Metab. 2009;9:311-26.

38. Ussher JR, Elmariah S, Gerszten RE, Dyck JR. The emerging role of metabolomics in the diagnosis and prognosis of cardiovascular disease. J Am Coll Cardiol. 2016;68:2850-70.

39. Bonsch D, Hothorn T, Krieglstein C, Koch M, Nehmer C, Lenz B, et al. Daily variations of homocysteine concentration may influence methylation of DNA in normal healthy individuals. Chronobiol Int. 2007;24:315-26.

40. Blanco RA, Ziegler TR, Carlson BA, Cheng PY, Park Y, Cotsonis GA, et al. Diurnal variation in glutathione and cysteine redox states in human plasma. Am J Clin Nutr. 2007;86:1016-23.

41. Forslund $A H$, Hambraeus $L$, van Beurden $H$, Holmback U, El-Khoury AE, Hjorth G, et al. Inverse relationship between protein intake and plasma free amino acids in healthy men at physical exercise. Am J Physiol Endocrinol Metab. 2000;278:E857-67. 
42. Breum L, Rasmussen MH, Hilsted J, Fernstrom JD. Twenty-four-hour plasma tryptophan concentrations and ratios are below normal in obese subjects and are not normalized by substantial weight reduction. Am J Clin Nutr. 2003;77:1112-8.

43. Schutz Y. Protein turnover, ureagenesis and gluconeogenesis. Int J Vitam Nutr Res. 2011:81:101-7.

44. White PJ, Newgard CB. Branched-chain amino acids in disease. Science. 2019;363:582-3.

45. Gar C, Rottenkolber M, Prehn C, Adamski J, Seissler J, Lechner A. Serum and plasma amino acids as markers of prediabetes, insulin resistance, and incident diabetes. Crit Rev Clin Lab Sci. 2018;55:21-32.

46. Locasale J, Dyar KA, Hubert MJ, Mir AA, Ciciliot S, Lutter D, et al. Transcriptional programming of lipid and amino acid metabolism by the skeletal muscle circadian clock. PLoS Biol. 2018;16:e2005886.

47. Zhenyukh O, Civantos E, Ruiz-Ortega M, Sánchez MS, Vázquez C, Peiró $C$, et al. High concentration of branched-chain amino acids promotes oxidative stress, inflammation and migration of human peripheral blood mononuclear cells via mTORC1 activation. Free Radical Biol Med. 2017; 104:165-77.

48. Harlan SM, Guo DF, Morgan DA, Fernandes-Santos C, Rahmouni K. Hypothalamic mTORC1 signaling controls sympathetic nerve activity and arterial pressure and mediates leptin effects. Cell Metab. 2013;17:599-606.

49. Han JM, Jeong SJ, Park MC, Kim G, Kwon NH, Kim HK, et al. Leucyl-tRNA synthetase is an intracellular leucine sensor for the mTORC1-signaling pathway. Cell. 2012;149:410-24.

50. Rahmouni K, Morgan DA. Hypothalamic arcuate nucleus mediates the sympathetic and arterial pressure responses to leptin. Hypertension. 2007;49:647-52.

51. Pawlak D, Tankiewicz A, Mysliwiec P, Buczko W. Tryptophan metabolism via the kynurenine pathway in experimental chronic renal failure. Nephron. 2002;90:328-35.

52. Nagy BM, Nagaraj C, Meinitzer A, Sharma N, Papp R, Foris V, et al. Importance of kynurenine in pulmonary hypertension. Am J Physiol Lung Cell Mol Physiol. 2017;313:L741-51.

53. Tengattini S, Reiter RJ, Tan DX, Terron MP, Rodella LF, Rezzani R. Cardiovascular diseases: protective effects of melatonin. J Pineal Res. 2008:44:16-25.
54. Pechanova O, Paulis L, Simko F. Peripheral and central effects of melatonin on blood pressure regulation. Int J Mol Sci. 2014;15:17920-37.

55. Long AN, Dagogo-Jack S. Comorbidities of diabetes and hypertension: mechanisms and approach to target organ protection. J Clin Hypertens (Greenwich). 2011;13:244-51.

56. Oliveras A, de la Sierra A. Resistant hypertension: patient characteristics, risk factors, co-morbidities and outcomes. J Hum Hypertens. 2014;28:213-7.

57. Yang G, Chu PL, Rump LC, Le TH, Stegbauer J. ACE2 and the homolog collectrin in the modulation of nitric oxide and oxidative stress in blood pressure homeostasis and vascular injury. Antioxid Redox Signal. 2017;26:645-59.

58. Zhao H, Liu Y, Li Z, Song Y, Cai X, Liu Y, et al. Identification of essential hypertension biomarkers in human urine by non-targeted metabolomics based on UPLC-Q-TOF/MS. Clin Chim Acta. 2018;486:192-8.

59. Wang $L$, Hou ET, Wang $L$, Wang $Y J$, Yang $L$, Zheng $X H$, Xie GQ, Sun Q, Liang MY, Tian ZM. Reconstruction and analysis of correlation networks based on GC-MS metabolomics data for young hypertensive men. Anal Chim Acta. 2015;854:95-105.

60. Harfmann BD, Schroder EA, Kachman MT, Hodge BA, Zhang X, Esser KA. Muscle-specific loss of Bmal1 leads to disrupted tissue glucose metabolism and systemic glucose homeostasis. Skelet Muscle. 2016;6:12.

61. Bass J, Lazar MA. Circadian time signatures of fitness and disease. Science. 2016;354:994-9.

62. Krishnaiah SY, Wu G, Altman BJ, Growe J, Rhoades SD, Coldren F, et al. Clock regulation of metabolites reveals coupling between transcription and metabolism. Cell Metab. 2017;25:1206.

63. Partch CL, Green CB, Takahashi JS. Molecular architecture of the mammalian circadian clock. Trends Cell Biol. 2014;24:90-9.

\section{Publisher's Note}

Springer Nature remains neutral with regard to jurisdictional claims in published maps and institutional affiliations.
Ready to submit your research? Choose BMC and benefit from:

- fast, convenient online submission

- thorough peer review by experienced researchers in your field

- rapid publication on acceptance

- support for research data, including large and complex data types

- gold Open Access which fosters wider collaboration and increased citations

- maximum visibility for your research: over 100M website views per year

At BMC, research is always in progress.

Learn more biomedcentral.com/submissions 